\title{
Economic and Technical Modeling of the Lebanese Crypto Currency: Implication for a Digital-Lira (DL)
}

\author{
Bassam Hamdar ${ }^{1}$, Tarek Saad ${ }^{2}$, \& Mohammad Hamdar ${ }^{3}$ \\ ${ }^{1}$ Department of Economics, Faculty of Business Administration, Al Maaref University, Beirut, Lebanon \\ 2 Department of Economics, Faculty of Business and Economics, American University of Science and Technology, \\ Beirut, Lebanon \\ 3 Department of Mathematics, Faculty of Arts and Sciences, American University of Beirut, Beirut, Lebanon \\ Correspondence: Bassam Hamdar, Dean, Department of Economics, Faculty of Business Administration, Al Maaref \\ University, Beirut, Lebanon.
}

Received: January 11, 2021

Accepted: February 18, $2021 \quad$ Online Published: February 23, 2021

doi:10.5430/ijba.v12n2p26

URL: https://doi.org/10.5430/ijba.v12n2p26

\begin{abstract}
Since 1997, the Lebanese pound has been pegged to the U.S dollar at a fixed rate, and at every crisis, people rush to banks to convert their LBP accounts into U.S.D causing a high demand for foreign currencies. Fear and uncertainty finds its way to the market, and the general sentiment often causes various sectors of the economy to be negatively affected. Lebanon is highly dependent on the U.S.D and the LBP had slowly become nothing but a symbol of independence and a heritage that tells the story of "Lebanon". This paper will examine the century's most aspiring technology to see how it can be implemented to create a new form of national currencies, and how such a technology can incorporate basic to complex monetary policies in an automated manner to gain value, and have a controlled inflation through a pre-programmed system. This paper will also break down the "Bitcoin" conditions and show how it can be modified to fit a locally produced national crypto-currency for Lebanon that will be referred to as "Digi-Lira". Finally, this paper will highlight the economic impact of the Digi-Lira on demand, investment, international trade, remittances, the unbanked population, and the banking sector.
\end{abstract}

Keywords: national crypto currency, digital-lira, economic modeling, Lebanon

\section{Introduction}

\subsection{An Overview of the Current Economic Situation in Lebanon}

What was once referred to, as the pearl of the Middle East, has now become a country on the verge of collapse. Lebanon that once attracted investors from around the world is now on its last breath. There is no single specific reason why the Lebanese economy is suffering, but rather, a series of events that added up to put Lebanon among the worst performing economies.

From the break of the Civil War in 1975, to the Israeli invasion, and the Syrian custody era, to the latest refugee crisis, Lebanon had always been firefighting various socio-political conflicts and this has led to bad political and economic decisions that were deemed necessary at certain times. The results of a long history of war and abrupt turmoil have created nothing but uncertainty in the Lebanese Economy.

What many fail to understand, is that, economic health is not just maintaining a proper exchange rate or even maintaining a healthy interest rate, an economy is a complex system where various aspects relate to one another to create a healthy system.

\subsection{A Historic Recapitulation of the USD Prominence}

Long before the United States joined World War II; it was the main supplier of weapons to the allies. Most of the payments done at that time were paid for in gold. By the end of WWII, the United States had become the holder of the vast majority of the world's gold reserve, dethroning Great Britain that found itself in debt for the very first time (John Milton Cooper, Jr. 1976). 
As the allies realized that their currencies could no longer be pegged to national gold reserves, it was decided during the Bretton Woods agreement (1944) that world currencies would be pegged to the USD, which was in turn pegged to its -very own - largest gold reserve (Investopedia 2020).

It was then that countries following WWII started accumulating USD reserves instead of gold, and consecutively the USD became the world's reserve currency.

Knowing that the majority of the countries following WWII were in debt to the US, it was no surprise that the latter had already established businesses and expanded its economic activities in almost every country. This fact was enough to crown the United States as "World's Largest Economy".

As a matter of fact, the Economic Growth of the United States was so prominent that even when the US treasury was issuing government securities to other countries to finance its war on Vietnam, way above its gold reserves, it was able to maintain the USD trust.

\subsection{Need of the Study}

Could the USD bullying practices and unprecedented prominence be the reason that countries such as Malta, Venezuela, Turkey, Iran, Russia, and several other European countries are embracing Bitcoin and other Blockchain based crypto currencies, or to say the least trying to develop their own? What would happen if countries extract themselves from the current economic system, and move into a new era of decentralized digital money built on the latest state of the art technologies, while still adhering to basic economic concepts?

\subsection{Problem Definition}

The Subject of USD prominence is vast and one could dwell on it. However, it is inevitably true to say that the level of control the US had been practicing in the past few years is unprecedented and things certainly need to change.

The USD is slowly but surely going to lose its prominence either to a new form of world currency (such as Bitcoin), or by having several world currencies serving as world reserves (i.e. Chinese Yuan, and Euro).

\subsection{Scope of the Study}

The study will examine this century's most aspiring technology to see how it can be implemented to create a new form of national currencies, and how such a technology can incorporate basic to complex monetary policies in an automated manner to gain value, and have a controlled inflation through a pre-programmed system. Moreover, this study will shed light on the Blockchain technology, and show how it provided solid grounds for the first use case of "Bitcoin". Therefore, this study will examine how the "Bitcoin" was created and dig deeper into the core of "Bitcoin" transactions, the so-called "Consensus mechanism". Finally, the authors will be breaking down the "Bitcoin" conditions and show how it can be modified to fit a locally produced national crypto-currency for Lebanon that will be referred to as "Digi-Lira".

\section{Literature Review}

\subsection{Overview}

Trust is a highly underappreciated term in economics. All national and international transactions revolve around this keyword. It is the banks, the governments, the financial institutions, and all other intermediaries that we "Trust" our money with to conduct basic transactions.

\subsection{Trusting the Wrong Entities}

The trust has always revolved around big players, be it a nation -where trust is given to a specific currency that has international recognition- or institutions such as international banks. These "trustees" have gained the upper hand on almost every transaction that goes around in the world, and they certainly are in control. Not only that, but the type of trust that was put in traditional systems has actually become a threat to us, both as individuals, and as citizens of different countries whose economies depend on the sole "likeness" of one prominent nation and its currency, the USD.

\subsection{Decentralizing Trust}

The idea of decentralizing trust is not new. People have depended on decentralized trust ever since online reviews and "airbnb" came to exist, but to talk about decentralized trust in a digital monetary system; it is all new to most of us.

"Trust" has, and will always be the key property of any transaction. People are willing to pay fees for a "trustable" entity to take care of their money. Same thing applies on a macro level. Governments want to transact through a "trustable" medium of exchange, a trustable entity. This is exactly how the banking system and central banks came to life. They play the role of a "Trustee". 


\subsection{Understanding Blockchain Technology}

The question of how to move from a "Centralized Trust "to a "Decentralized trust is tackled by the "peer to peer" payment solution that is based on "Blockchain Technology".

Traditionally, almost every monetary transaction is added into a ledger that holds all historic transactions from and to a specific account. These ledgers are trusted into just one or a few parties (usually banks and other financial institutions) that keep records of all transactions. However, there is no way to know whether or not this party is altering or adding any invalid transactions into their ledgers, after all they are the sole owner of the ledgers and access to information is very limited. These kinds of ledgers are usually "Private" and "Centralized".

In Blockchain technology, things are very different. In a Blockchain based project, the ledgers are "public", "decentralized" and "tamper proof". Every single human being that has Internet access can publicly view the ledgers on the Blockchain. This property plays an important role in providing transparency to the system.

In addition, any transaction that goes into a Blockchain ledger is not approved by a single entity, but rather by the power of a large collective network of computers that compete to complete this transaction and verify its validity, while also, giving it value.

\subsection{Proof of Work (POW) Consensus Mechanism}

The act of making sure that transactions on a Blockchain ledger are trustable, valid and tamper proof is called "The Consensus Mechanism" and it is at the core of Blockchain technology.

In a "POW" consensus mechanism, transactions (payment orders or any data entry) on the Blockchain are bundled together in blocks of data. Each block of transactions is propagated to the network where miners (individuals using computing devices) compete (by employing computing power) to solve a complex mathematical algorithm that represents the given block of transactions (CasinoCoin Foundation, 2018).

Once the mathematical algorithm of a given block is solved, it is decrypted into a 64-digit hexadecimal code (known as a cryptographic hash or a digital fingerprint that acts as a reference) and is communicated to the rest of the network. This cryptographic hash is of utmost importance to the "POW" consensus mechanism since it acts as a link between newly and previously solved blocks, thus creating an "Immutable" Blockchain ledger that is tamper-proof.

\subsection{Bitcoin}

Bitcoin was the first application of a POW consensus mechanism using Blockchain. It is the crypto currency that miners receive in return for deciphering the block (mining a block).

Let us look at the Bitcoin Transaction Lifecycle. The technical workflow process is utilized to explain earlier using the bus station example:

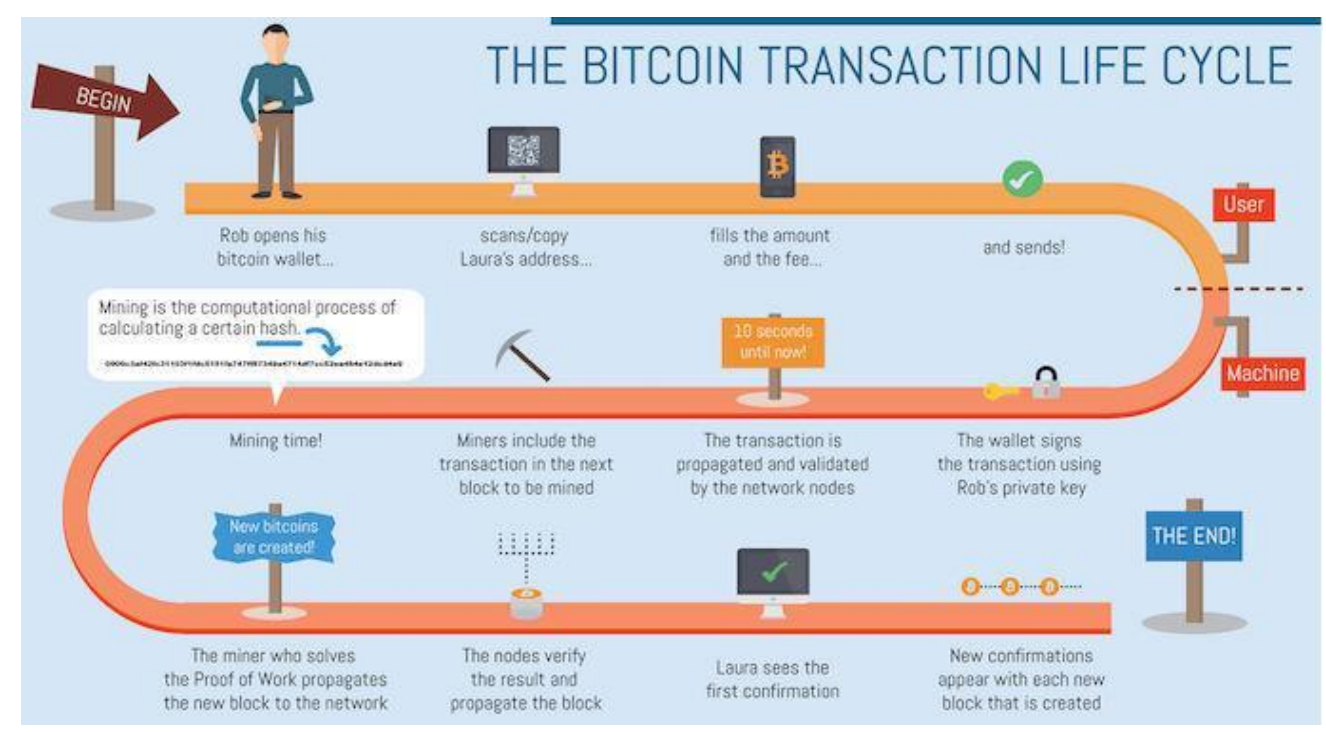

Figure 1. The bitcoin transaction life cycle 
As it can be shown from the above figure, if Bob wants to send \$100 USD worth of Bitcoin to Laura, he opens his wallet, scans Laura's Bitcoin wallet address, fills the amount he desires to send, and generates the process.

The order to pay leaves Bob's Bitcoin wallet and Bob's transaction rides the next "Block of Transactions" (remember blocks loads transactions and leave the station every 10 minutes).

Once Bob's transaction gets in a block and it is time to leave the station, that whole block is then propagated to the network where miners compete by using high computing power to unlock the complex mathematical algorithm protecting the block. Once deciphered, the block will propagate the obtained 64- digit hexadecimal code to the whole network. The network will examine and confirm that the code is correct. It does so by examining individual transaction histories of all the transactions contained in the block. It makes sure that indeed, Bob has enough money to send, and that true, his destination is Laura's wallet. This process happens automatically to all transactions, within the block in such a way that if one miner (gatekeeper) tries to manipulate a single transaction and hamper the block's safety, the system will throw the miner out of the network, and whatever block one tries to manipulate, it will be deleted, and replaced by the true verified block of transactions.

\subsection{Introducing the Digi-Lira}

As of the date of this study, the creator of Bitcoin is unknown but goes with the pseudonym of "Satoshi Nakamoto". The creator may be a single person or a group of people who have successfully created a digital currency, which was first, introduced in the market in 2009 at a price of three US cents, and has ever since gained more and more global acceptance reaching an all-time high of $\$ 20,000$ for one Bitcoin in late 2017, and approximately $\$ 50,000$.

If an anonymous person can create such a digitally sound form of money that has most of the economic concepts integrated into it in an automated manner, why cannot governments learn from this breakthrough in Financial Technology (Fintech), and create a new form of digital currencies?

This study highlights the feasibility of creating a new national currency in Lebanon, "Digi- Lira". The "Digi - Lira" would be built on a modified code of Bitcoin, while keeping in mind all the technical and economic concepts that Bitcoin entails.

\section{Methodology}

\subsection{Qualitative Content Analysis}

To better understand how to formulate the "Digi-Lira", it is needed to dive deeper into the constituents of the Bitcoin system that drives its value from both technical and economic perspectives.

\subsection{Frequency of Blocks}

As mentioned earlier, transactions on the Bitcoin network are bundled in blocks before being propagated to the network for miners to decipher and verify.

In order not to disrupt or overload the system, a new block of transactions is only propagated to the network every 10 minutes, allowing enough time for the miners to solve the complex algorithm (deciphering) of the previous block, and validate it (verifying transactions). A block may contain just one or several transactions (up to two MB of transactions) (Stewart Fortier 2018).

\subsection{Mining Difficulty}

It is important to note that the difficulty of the mathematical algorithm that protects a block of transactions adjusts every 2016 blocks $(2016 / 144$ blocks per day $=14$ days). This means that every 14 days, the system tests the computing power of miners on the network, and accordingly, adjusts the difficulty of the complex mathematical algorithm that protects the blocks ( Ankalkoti, 2017).

The mathematical algorithm:

Computers use binary language to communicate. This language is long and complex. Any type of data can be converted into a binary string; in turn, the binary string can be translated into a much shorter hexadecimal code that accurately represents the binary code and therefore represents the data.

Example;

The word "Hello" in binary translates to:

“01101000 $01100101011011000110110001101111 ”$ (40 characters)

While in hexadecimal, it translates to: 
“68 65 6c 6c 6f” (10 characters).

Now because the hexadecimal code gets longer as the binary input increases, computer scientists came up with what is currently known as a "Hash Function".

"Hash Function".

A hash function takes any binary input, no matter how short or long it is, and translates it into a "Fixed" length hexadecimal code.

In this manner, the hashed function of the Letter " $A$ " can be represented in a 64 digit hexadecimal string, much as if all the works of William Shakespeare combined can also be represented in a 64 digit hexadecimal string.

What differs between hashing the letter "A" and all the works of "William Shakespeare" combined is the hashing power required to obtain that "fixed" length hexadecimal string, i.e. the difficulty.

Hashing power is therefore the rate at which a computing device can process a number of hashes per second.

- $\quad$ CPU (the regular computer): Depending on what CPU is being used; the hashing power may vary from $1.4 \mathrm{Mh} / \mathrm{s}$ (Mega hashes/second) up to $15.8 \mathrm{Mh} / \mathrm{s}$.

- $\quad$ GPU (High Processing graphic cards): GPU's hashing power may vary from $11 \mathrm{Mh} / \mathrm{s}$ up to $64 \mathrm{Mh} / \mathrm{s}$.

- $\quad$ ASICs (Application Specific integrated Circuit): ASICs hashing power may vary from $800 \mathrm{Mh} / \mathrm{s}$ up to five Gh/s (Giga hash per second).

- $\quad$ Antminer S9: Antminer S9 is among the highest hashing power processors at $14 \mathrm{Th} / \mathrm{s}$ (Terra hashes per second).

As the complexity of data to be hashed into a "fixed" hexadecimal string increases, so does the need for a higher hashing power? (Harsh Agrawal 2019).

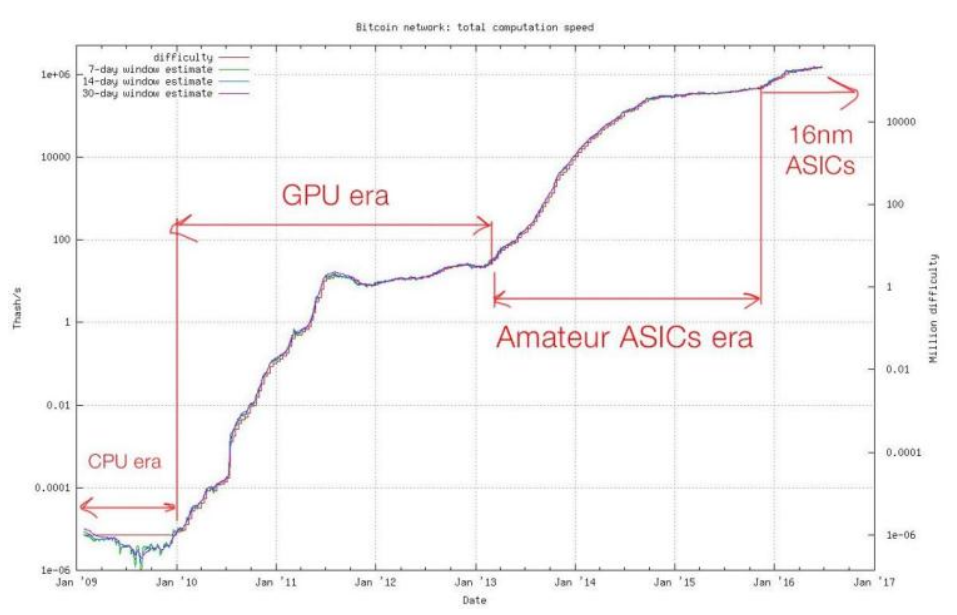

Figure 2. Mining difficulty

The above graph does not only show how many people willingly employed resources to mine Bitcoin blocks, but it also is a great indicator of the increasing demand on Bitcoin.

\subsection{Mining Reward}

As mentioned earlier, in the "Proof of Work" consensus mechanism, which Bitcoin was built on, the miner (or group of miners) who solves the complex mathematical algorithm is rewarded with a crypto currency - in this case a batch of Bitcoins.

What is notable here is the fact that Bitcoin's mining rewards decrease at the rate of $50 \%$ (halving) every 210,000 blocks (4 years).

The first halving of Bitcoin reward per block occurred in November 2012 where Bitcoin rewards dropped to 25 Bitcoins per block; doing the same math, the period between 2012 and 2016 yielded 5,256,000 Bitcoins (25\% of total supply) (Conway, 2020). 
To sum up, as more blocks are mined the mining difficulty seems to be increasing. Miners are co-working in mining pools resulting in higher hashing power being employed. This in turn is causing a higher difficulty adjustment to occur most of the times. Simultaneously, the block rewards are decreasing.

\subsection{How Can Blockchain Technology Revamp the Lebanese Economy?}

The uses of Blockchain technology in any economy are endless. Because of its decentralized trust and tamper proof properties, several countries have implemented Blockchain technologies into areas such as "Voting Systems", "Utility Bills", "Imports and Exports Records", "Medical Fields", "Travel Industry", and much more.

For instance, Venezuela issued a "Petro Coin" claiming that it is backed up by Venezuela's Oil reserves. The Petro Coin was sold in the market as an ICO (Initial Coin Offering).

\section{The Economic Approach to Digi-Lira}

\subsection{Phasing Out the "Digi-Lira"}

This study proposes a mineable Lebanese crypto currency issued by the Lebanese central bank in collaboration with Lebanese commercial banks and the citizens of the country.

A- 2 phase approach that starts with creating value through the mining of a "Digi-Lira", and ends by changing the protocol once the last coin is mined, giving it a fast transacting property with minimal fees.

\subsection{Phase 1}

The "Digi-Lira" would be the new Lebanese crypto currency built on a modified code of Bitcoin for all the reasons explained in this study.

Just as Bitcoin, the "Digi-Lira" would be a crypto currency that acts as a commodity in its initial phase; meaning is not meant for everyday use, rather, it acts as a store of value that can also be traded.

\subsection{Phase 2}

Because the mining phase is essential in creating value, moving to Phase 2 will only occur once the last "Digi-Lira" is mined.

In Phase 2, the rewards will cease, and the adjustment difficulty will update every hour to adjust into a much lower block interval.

At that point, transaction fees from all transactions in a block would be equally distributed to miners who would at that point be acting as validators.

\subsection{Igniting Demand}

Igniting demand on the "Digi-Lira" and achieving local and regional acceptance is of utmost importance. It is understood that achieving such acceptance requires immense effort on educating citizens and businesses on the technology of Blockchain, and how digital currencies gain value from the Proof of Work process. The following are necessary drivers of demand.

\subsection{Mining Demand}

One of the essential triggers for "Digi-lira" demand would be the fact that mining should be open to the public following the first $50 \%$ acquisition by the central bank of Lebanon. It is important for the central bank of Lebanon to hold more than $50 \%$ of the total supply in order to initiate higher volumes of transactions.

\subsection{Attracting Investors}

Since the "Digi-Lira" would be initiated and approved by the central bank of Lebanon, and has a controlled supply scheme resulting in a higher future value, it is only normal to expect a high appeal from investors in both private and public sectors. Buying the "Digi-Lira" would only be possible through the Lebanese Pound or Lira for local investors. This will increase demand on the "Digi-Lira" while also increasing demand on the Lebanese Lira.

\subsection{International Trade}

The government has to support the "Digi-Lira" by employing rules and regulations on international trades that favors the use of "Digi-Lira".

Some of the benefits of using Digi-Lira in international trade are:

1- Direct Banking with the central bank of Lebanon without the need for routing with international banks (Specific to sanctioned economies) 


\section{2- Lower Tariffs}

\section{3- Lower Taxation}

\subsection{Unbanked Population}

One of the big challenges facing Lebanon is the number of unbanked people, be it Lebanese citizens, or even refugees. This results in an increasing number of money being traded internally and externally beyond the supervision of the central bank.

The central bank of Lebanon would appoint specialized centers that issue "Digi-Lira" electronic wallets for such people with minimum verification documents. These centers would also facilitate conversion of paper money into the "Digi-Lira".

\subsection{Remittances, Commercial Use, and the Banking Sector}

The Lebanese diaspora will certainly find it more convenient to send money to families in Lebanon using the "Digi-Lira", Instead of going through the regular international transfers.

Once phase 2 commences, transacting with the "Digi-Lira" would be fast and efficient. Commercial use of "Digi-Lira" would be made available as more stores and outlets start using and integrating it into their Point Of Sale (POS's). In addition, by that time, "Digi-Lira" ATMs would be available at every corner to either withdraw or deposit fiat currency.

The Lebanese banking sector is one of the strongest sectors in the Lebanese economy and it should not be disrupted. Banks might benefit in several ways from the "Digi-lira"

1- Banks may employ their resources to help mine the "Digi-Lira"

2- The central bank of Lebanon may allow commercial banks to build upon the "Digi-lira" e-wallet in a way that additional services are provided to people using the bank's e-wallet.

3- A person may choose to store his/her "Digi-lira" in a bank and earn returns the same way a saving account works.

4- A bank may issue personal loans and credit based on a person's "Digi-Lira" holding in their wallet.

\section{Conclusion and Recommendations}

\subsection{Overview}

Discussing a new form of currency, one that is built on a new kind of technology that very few people understand in developing countries is certainly a big challenge. Blockchain technology can be integrated in almost any industry where the need for trust and fast transacting is essential.

\subsection{Conclusion}

Most international trade transactions are concluded in USD, as it is the world most trusted currency. However, as discussed previously, this has given the USA an upper hand over all other nations allowing it to place sanctions and practice political bullying on countries that do not adhere to the U.S agenda.

This could change if each country is able to create a new decentralized sovereign currency based on the Blockchain technology. However, to do so, one may think that a decentralized world currency to use as a medium of exchange is needed. This decentralized world-currency already exists - "Bitcoin".

Iran, Russia, and Venezuela are some of the first countries that have started accumulating Bitcoins as part of their foreign currency reserves. The use of cryptocurrency is expected to stimulate domestic demand, increase consumption, facilitate international trade, attract investment, involve the unbanked population, benefit the banking sector, and through the smoothing out of the remittance process, savings and investments will be encouraged.

\subsection{Recommendation}

To achieve this kind of liberation, countries would first need to reconsider traditional economics and be open, with a certain amount of doubt, to new technologies that can unleash endless possibilities or even perhaps fundamentally change what was once considered impossible.

Blockchain technology does not have to revolve around a sovereign currency; however, at the core of Blockchain, is a costly process that rewards participants to become decentralized, which can be used to fight corruption by enforcing laws that provokes the use of decentralized applications. 
Developing Countries can save billions of dollars by fighting corruption through Blockchain technology. Imagine what would happen if all transactions (from maritime port trading, to customs, and tax systems) are executed on an immutable ledger that could not be tampered with or hacked. Governments will have full transparency of all transactions, and therefore trace back any transaction to its root with full visibility.

\section{Limitations}

Understanding how many "Digi-Liras" the system would produce, and how long the roll out phase should be is a real limitation. Another limitation in the proposed scheme is the adjustment difficulty required to mine the next block of transactions for the "Digi-Lira", and the computational power it may require as well as the availability of infrastructure to support such requirements.

Finally, another limitation is the fact that prices of crypto currencies are based on supply and demand of such currencies, which may lead to hyperinflation or deflation.

\section{Future Research}

According to the World Gold Council, Lebanon is at the $16^{\text {th }}$ rank with 286.8 metric tons of gold reserve.

A pre-mined crypto currency backed by a gram of gold with a total supply equal to the total gold reserve in Lebanon, should be discussed and considered as a basis for the future research in crypto currency backing strategies. This would work perfectly and revive the gold standard in a digital way; however, this will eliminate the value that the currency would gain because of increasing demand, since the new currency price would be solely linked to the price of gold. Alternatively, Lebanon will soon be producing Oil. Another option would be to create a pre-mined crypto currency backed by Oil. However, as the Oil prices are greatly affected by world events such as sanctions, war, and OPEC resolutions, the coin price will not have the needed Stability.

\section{List of Abbreviations:}

POW: Proof of Work

Mb: Mega byte

Mh/s: Mega Hash per Second

Gh/s: Gega Hash per Second

Th/s: Tera Hash per Second

CPU: Central Processing Unit

ASIC: Application Specific Integrated Circuit

LDMP: law of Diminishing Marginal Product

MC/MP: Marginal Cost to Marginal Product

AMST: Asymptotic Money Supply Targeting

GDP: Gross Domestic Product

ICO: Initial Coin Offering

BDL: Banque De Liban

LL: Lebanese Lira or Lebanese Pound

OPEC: Organization of Petroleum Exporting Countries

\section{Key Terms:}

\section{1- Crypto Currency:}

A digital currency that is produced using Block Chain technology.

2- Bitcoin:

The first crypto currency produced using Block Chain technology.

3- Block Chain Technology:

A Block Chain is a decentralized, distributed and public digital ledger that is used to record transactions across many computers so that any involved record cannot be altered retroactively, without the alteration of all subsequent blocks.

4- Gold Standard: 
A term used in reference to pegging a national currency to its equivalent in gold reserves.

5- Digi-Lira:

A name given to the crypto currency proposed in this study.

6- Megabyte/ Gigabyte/ Terabyte:

Are data measurement units applied to digital computer or media storage.

7- Proof of Work (POW):

A consensus mechanism that drives the value of a Block Chain technology by employing digital labor and capital.

8- Consensus Mechanism:

A process in which a network consisting of computational power reaches agreement on the validity of data added to a ledger and rejection to faulty alterations.

9- Hexadecimal Code:

Is a positional numeral system with a radix, or base, of 16 , It uses sixteen distinct symbols, most often the symbols "0"-"9" to represent values zero to nine, and "A"-"F" (or alternatively "a"-"f") to represent values ten to fifteen.

10- Cryptographic Hash:

Is a special class of hash function that has certain properties, which make it suitable for use in cryptography. It is a mathematical algorithm that maps data of arbitrary size to a bit string of a fixed size (a hash) and is designed to be a one-way function, that is, a function which is infeasible to invert.

11- Cryptographic Block:

Is a deterministic algorithm operating on fixed-length groups of bits, called a block, with an unvarying transformation that is specified by a symmetric key. It operates as an important elementary components in the design of many cryptographic protocols, and are widely used to implement encryption of bulk data.

12- Mining:

Is the process of employing computational power to solve a cryptographic hash function.

13- Binary:

A computer language of interpreting data in term of 0's and 1's.

14- Antminer s9:

Is a type of ASIC's and the world's most powerful Bitcoin miner, which is smaller in size than many other portable boom boxes available.

15- Mining Farm:

Is a data center, technically equipped to mine Bitcoins or other crypto currencies.

\section{References}

Agrawal, H. (2019). Explaining hash rate or hash power in crypto currencies. Retrieved from www.coinsutra.com

Ankalkoti, P. (2017). A Relative Study on Bitcoin Mining. Imperial Journal of Interdisciplinary Research (IJIR). Retrieved from https://www.researchgate.net/publication/318850089_A_Relative_Study_on_Bitcoin_Mining

Bank of England. (n.d.). Why does money depend on trust?. Knowledge Bank Report. Retrieved from https://www.bankofengland.co.uk/knowledgebank/why-does-money-depend-on-trust

Bitcoin/ Hashrate Chart. (2021). Average hashrate (hash/s) per day. Bitcoin Hashrate historical chart. Retrieved from https://bitinfocharts.com/comparison/bitcoin-hashrate.html

Bordo, M. D. (2008). Gold Standard. Econlib's Economic History collection. Retrieved from https://www.econlib.org/library/Enc/GoldStandard.html

Buy Bitcoin Worldwide. (2021). How many bitcoins are there? Retrieved from https://www.buybitcoinworldwide.com/how-many-bitcoins-are-there

Chenn, J. (2020). What is a reserve currency?. Investopedia. Retrieved from https://www.investopedia.com/terms/r/reservecurrency.asp 
Consensus: The Core of blockchain Technology. (2018). Casino coin foundation. Retrieved from https://casinocoin.org/consensus-the-core-of-blockchain-technology

Conway, L. (2020). Bitcoin halving. Retrieved from https://www.investopedia.com/bitcoin-halving-4843769

Cooper, J. (1976). The Command of Gold Reversed: American Loans to Britain, 1915-1917. Pacific Historical Review, 45(2), 209-230. https://doi.org/10.2307/3638495

Eric Le, B., \& Jacobs, T. J. (2016). Lebanon: promoting poverty reduction and shared prosperity. October Review, Lebanon's Economic Outlook, World Bank Group.

Fortier, S. (2018). Proof-of-work (for dummies). Retrieved from https://medium.com/coinmonks/proof-of-work-for-dummies-5abad1cd744

Jihad El, H. (2018). Nharkom Said -LBCI. Retrieved from https://www.lbcgroup.tv/watch/30428/jihad-el-hokayem/en

Katzman, K. (n.d.). Iran Sanctions. Congressional Research Service RS20871. Retrieved from https://fas.org/sgp/crs/mideast/RS20871.pdf

Lebanon's Economy Has Long Been Sluggish Now A Crisis Looms. (2018). The Economist. Retrieved from https://www.economist.com/middle-east-and-africa/2018/08/30/lebanons-economy-has-long-been-sluggish-now -a-crisis-looms

Lopp, J. (2015). The future of bitcoin mining. Cypherpunk Cogitations. Retrieved from https://medium.com/@lopp/the-future-of-bitcoin-mining-ac9c3dc39c60

Majaski, C. (2020). Distributed ledgers. Retrieved from https://www.investopedia.com/terms/d/distributed-ledgers.asp

Rochard, P. (2013). The bitcoin central bank's perfect monetary policy. Satoshi Nakamoto Institute. Retrieved from www.nakamotoinstitute.org

\section{Copyrights}

Copyright for this article is retained by the author(s), with first publication rights granted to the journal.

This is an open-access article distributed under the terms and conditions of the Creative Commons Attribution license (http://creativecommons.org/licenses/by/4.0/). 